\title{
Yes, More Decoherence: A Reply to Critics
}

\author{
Elise M. Crull*
}

Submitted 11 July 2017; revised 24 August 2017

\section{Introduction}

A few years ago I published an article in this journal titled "Less interpretation and more decoherence in quantum gravity and inflationary cosmology" (Crull, 2015) that generated replies from three pairs of authors: Vassallo and Esfeld (2015), Okon and Sudarsky (2016) and Fortin and Lombardi (2017). As a philosopher of physics it is my chief aim to engage physicists and philosophers alike in deeper conversation regarding scientific theories and their implications. In as much as my earlier paper provoked a suite of responses and thereby brought into sharper relief numerous misconceptions regarding decoherence, I welcome the occasion provided by the editors of this journal to continue the discussion. In what follows, I respond to my critics in some detail (wherein the devil is often found). I must be clear at the outset, however, that due to the nature of these criticisms, much of what I say below can be categorized as one or both of the following: (a) a repetition of points made in the original paper, and (b) a reiteration of formal and dynamical aspects of quantum decoherence considered uncontroversial by experts working on theoretical and experimental applications of this process. ${ }^{1}$

I begin with a few paragraphs describing what my 2015 paper both was, and was not, about. I then briefly address Vassallo's and Esfeld's (hereafter VE) relatively short response to me, dedicating the bulk of my reply to the lengthy critique of Okon and Sudarsky (hereafter OS). The contribution to this debate by Fortin and Lombardi (hereafter FL) largely substantiates my points; I will call upon their paper at various times in my defense.

\footnotetext{
${ }^{*}$ The City College of New York, CUNY. ecrull@ccny.cuny.edu

${ }^{1}$ This latter point explains in part why I did not include equations in the original paper - a fact that Okon and Sudarsky mention more than once as a defect of my account. I had assumed (wrongly, it seems) that the basic aspects of decoherence were well enough known and appreciated from the prodigious and respected literature - much of which I cited in my paper - that instead of presenting it all yet again, I might more profitably focus on certain philosophical aspects. I will try to remedy this lack of formalism to my critics' satisfaction in what follows, but again I direct the reader to references cited in this and the original paper.
} 


\subsection{What my paper was about}

The aim of my original paper was to urge researchers investigating applications of quantum theory (especially, but not exclusively, relativistically) to consider the fruitfulness of decoherence studies and incorporate these results in their own pursuits. Doing so, I argued, would have several benefits. It would significantly delay the moment when various interpretations of quantum mechanics, on which there is no consensus, were called upon to explain certain dynamical facts. This thereby lessens the significance of betting on the wrong horse (or indeed on any one horse) in a race of indeterminate length. ${ }^{2}$

My motivation for presenting a breakdown of the measurement problem similar to that of Max Schlosshauer in his textbook on decoherence (Schlosshauer, 2007) was to make clear that while decoherence cannot provide an answer to the question of why one specific, apparently determinate outcome is measured rather than another, it can provide satisfactory explanations for kindred questions like the selection of preferred bases and the lack of observable superpositions. With these explanations forming my premises, I argued that troubles encountered within relativistic applications of quantum theory frequently attributed to the measurement problem stem from those aspects of the problem decoherence in fact explains. Hence, progress can be made despite the intractability of the problem of specific measurement outcomes.

\subsection{What my paper was not about}

Nowhere in the original paper was it said that interpretations of quantum mechanics could be eliminated altogether (pace OS and VE), nor that decoherence solves all problems to completeness (pace FL); my claims were more tempered than that. Regarding the latter, FL understood me to be offering a too-rosy view of decoherence as a concept free from controversy and perfectly well understood. Certainly not. But it should be involved in far less controversy than it is, and what is more, what the consensus view consists in is sufficient physics to run my arguments.

Perhaps these misunderstandings arose because the claim has been made elsewhere (though never by me) that decoherence solves the measurement problem tout court. However, since in the original paper I explicitly denied this incorrect view of decoherence, I am unsure what to say to my critics. Likewise, perhaps some of the misunderstanding of my points was due to the widespread and persistent conflation of "decoherence theory" or "environment-induced decoherence", or simply "decoherence processes" - none of which exceed the implications of the bare quantum formalism - with explicitly interpre-

\footnotetext{
${ }^{2}$ Those who, prior to decoherence considerations, are already standard-bearers for a particular interpretation ought to acknowledge that decoherence importantly alters both the motivations for and implementations of the various candidate interpretations. However, cataloguing those differences is not my aim here, nor was it in the original paper. Instead, my aim was to emphasize that one can invoke decoherence processes from a standpoint that is neutral with respect to the interpretation debate, and still gain significant explanatory benefits.
} 
tive projects that take decoherence (qua physical process) as their starting point, like the decoherent histories interpretation of Gell-Mann and Hartle (1996) and Halliwell (1995). But once again, in the original paper I was careful to place myself firmly in the former camp regarding my employment of the term "decoherence"; such misunderstandings are unnecessary.

\section{Reply to Vassallo and Esfeld}

VE raise two primary objections that overlap somewhat with comments made by OS; I will respond to them together here, as this will prove a useful starting point. The first objection concerns what precisely I meant by "the standard quantum formalism", and whether or not this requires assuming wave function collapse or the Born rule. The second objection concerns Schrödinger's cat.

\subsection{The Bare Formalism}

VE write: "While we agree with the author that decoherence is a powerful tool that effectively helps us dealing with the conceptual asperities of quantum physics - the measurement problem above all -, we do not see how this fact might dispense us with the urge to interpret the bare quantum formalism" (Vassallo and Esfeld, 2015, p. 1533). ${ }^{3}$

Here VE misunderstand my general point, which (as reiterated above) is not to say one can do away with interpretation entirely, but rather that one might profitably postpone the invocation of particular interpretations of quantum mechanics. Admittedly I ought to have been explicit about what I took the standard formalism to be. OS criticize both Max Schlosshauer (who, recall, literally wrote the book on decoherence) and myself on this point: "...by not stating precisely what is the concrete quantum formalism he [Schlosshauer] endorses, he allows for some 'wiggle room' at the moment of physically interpreting the formal results obtained by decoherence" (OS p. 858). Schlosshauer does in fact state in later chapters which aspects of the formalism he requires. I, on the other hand, need only the propositions OS themselves allow - but with important qualifications: the complete statistics of mixed states can be represented by density operators in a Hilbert

\footnotetext{
${ }^{3}$ Hereafter, unless otherwise noted, all references to VE are to this article; likewise, all references to OS are to Okon and Sudarsky (2016) and all references to FL are to Fortin and Lombardi (2017).
} 
space, ${ }^{4}$ observables ("properties") are typically self-adjoint operators, ${ }^{5}$ and the Schrödinger equation describes the unitary evolution of closed systems. Add to these three propositions certain widely accepted facts about the superposition rule, entanglement, and the ubiquity of quantum interactions (conversely, the nonexistence of a truly closed system aside from the universe as a whole), and one has enough to get decoherence dynamics.

I disagree with OS when they claim that I cannot arrive at decoherence without also assuming wave function collapse and the Born rule (ergo the eigenstate-eigenvalue link). This is not the case, though why I do not require these assumptions understandably becomes murky owing to OS's conflation of "standard formalism" with "standard interpretation" at various times in their exposition (where the latter is meant to refer to von Neumann's view or the mythical Copenhagen interpretation, perhaps? Neither of which, please note, I endorse).

Regarding the question of collapse, VE and OS are certainly not the first to elide components of physical collapse theories like GRW with the effects of decoherence. In fact, this mistake is so frequent that the Stanford Encyclopedia of Philosophy entry on decoherence contains a caveat listing two physical assumptions that collapse theories require but that decoherence does not. It will be instructive to repeat them here. First, collapse theories require true, physical collapse of the wave function; decoherence only entails effective collapse, in that this process renders practically impossible measurement results that are not eigenstates of an unperturbed basis (Bacciagaluppi, 2012). The experimental fact that certain decohered systems exhibit coherence revivals demonstrates that no physical collapse occurred in these cases. ${ }^{6}$

The second assumption collapse theories require but decoherence does not is the existence of an as-yet undiscovered collapse mechanism that introduces nonlinearity apart from the system-environment Hamiltonian. Decoherence introduces no additional nonlinearity but "simply takes real, inevitable, interaction with external degrees of freedom to bear in the Hamiltonian" (Bacciagaluppi, 2012). To this add still a third, related point conceded by collapse theorists themselves. Because collapse theories do not explain the privileging of

\footnotetext{
${ }^{4}$ Qualifications: (i) mixed states needn't be represented this way; e.g., one might equally well employ the reduced Feynman path integral (RPI) approach described in Mensky (2000). Also, (ii) while both proper and improper mixtures may have identical density matrices, they nevertheless represent vastly different physical situations: the former represents a classical statistical distribution of possible states (where the system definitely occupies one state but it is unknown which), while the latter represents an entangled case (where the system cannot be said to occupy a single state from the ensemble). Because entanglement is a necessary condition for decoherence, whenever one uses the density matrix formalism to study decoherence processes, one is dealing with improper mixtures. One must not lose sight of this failure of uniqueness in the formalism.

${ }^{5}$ Qualifications: again, this proposition isn't strictly necessary within the standard formalism. Observables - or operators more generally - are derivative entities used to link the formalism to the empirical data. This point was made in the original paper in discussion of Shimony's epistemic circle and by referring to research conducted using non-Hermitian operators.

${ }^{6}$ See Narozhny et al. (1981) for theoretical groundwork and Kokorowski et al. (2001) as an entry point into fascinating work verifying decoherence models through experiments in atomic interferometry.
} 
certain bases, this piece of the dynamics is explained by decoherence. In other words, that decoherence is not an interpretation of quantum mechanics nor does it require anything beyond the bare formalism is substantiated by the fact that all viable candidate interpretations include decoherence as part of their explanatory package. To wit, a comment made in a recent paper discussing the de Broglie-Bohm $[\mathrm{dBB}]$ interpretation:

The empirical adequacy of dBB theory does rely on decoherence, since as Bohm showed (Bohm, 1952b), decoherence serves as the lynchpin for the theory's effective collapse mechanism; without decoherence, the apparatus configuration in the measurement does not become irreversibly correlated to the measured system, nor does the theory recover the Born Rule for measurements of nonposition observables. (Rosaler, 2015, p. 1186)

In summary, no physical collapse is needed so long as one stops short of attempting to answer the specific problem of outcomes, as I did in the original paper. Effective wave function collapse is needed to explain preferred bases, but more on this shall be said below.

Similar reasoning applies to Born's rule and the eigenstate-eigenvalue link: as long as one stops short of declaring the results of measurements to be actually definite - which is a giant ontological step beyond declaring the results of measurements to be only apparently definite - then the Born rule can be understood as merely a guide to expectations and not a metaphysical claim requiring further proof. David Wallace explains this nicely in a recent book (Wallace, 2012b, p. 22):

Readers familiar with typical discussions of the measurement problem may be surprised that I have mentioned neither the "eigenstate-eigenvalue link" nor the "collapse of the wavefunction". This is deliberate: neither plays any role in the formalism of quantum mechanics, and neither plays any role in the algorithm by which we can (pragmatically speaking) extract empirical predictions from quantum mechanics. And neither plays any role in modern discussions of measurement in mainstream quantum physics.

He expands on these comments in a footnote:

The former rule [eigenstate-eigenvalue link] plays no role in calculations, serving only to ground the metaphysical supposition that "measurements" reveal some pre-existing value, and to ground it only in certain special circumstances; it is sometimes erroneously thought to link the quantum state to our observations in "unproblematic" cases where there is no macroscopic superposition, but it universally fails to do so at least in the case of position or momentum (no physically realistic quantum system's wavefunction remains bounded in a finite spatial region for more than an instant). The latter rule [collapse of the wavefunction] superficially seems necessary to account for repeated measurements, but in fact those measurements are invariably handled within unitary 
quantum theory... The obsolescence of both rules is clear from the fact that they cannot even be defined in cases where a POVM, rather than a PVM, is used to represent the measurement process.

A slightly fuller version of the arguments sketched here can be found in Wallace (2012a), "Decoherence and its role in the modern measurement problem". The takeaway from these arguments and my own are that one need not attribute ontological meaning to the Born rule as long as one avoids assuming that appearances depict reality, that measurement outcomes are all and only what they seem.

\subsection{Schrödinger's Cat}

VE's other main criticism of my thesis involves employing decoherence processes to explain Schrödinger's cat. ${ }^{7}$ FL discuss the question of Schrödinger's cat in their reply, bringing this well-worn example into a broader discussion of open versus closed systems. For my part I defer to the abundant experimental literature involving decoherence processes and observed Schrödinger cat states, beginning with the work of Sergè Haroche and colleagues. Haroche was awarded the 2012 Nobel Prize in Physics for his work on entangled photons and atoms (sometimes called "Schrödinger's kittens") in an optical cavity. In his Nobel Prize lecture, he described his work as follows:

Letting a coherent field evolve under its coupling with a Rydberg atom at resonance turns the atom-field system, after some time, into an entangled atomfield state superposition involving two coherent fields with opposite phases. The two components of this cat merge together at a later time. This effect of the field phase splitting and recombination is related to the collapse and revival of the Rabi oscillation phenomenon. The Schrödinger cat experiments in Cavity QED illustrate the fragility of quantum coherences in systems made of increasing numbers of particles. They give us a glimpse at the boundary between the quantum world, where state superpositions are ubiquitous, and the classical one. (Haroche, 2012, pp. 90-91)

Similar experiments are reported in Bernu et al. (2008), Bertet et al. (2002), Davidovich et al. (1996), Deléglise et al. (2008), Raimond et al. (2001) and Raimond and Haroche (2005). Let us consider more carefully a study of this type titled "Scheme to probe the decoherence of a macroscopic object"(Bose et al., 1999). The authors model a cavity field as the microscopic system and a movable mirror as the macroscopic system (the cat). At the beginning of their report, they explain the limitations of a "true" Schrödinger cat experiment, but go on to address the concern VE raise - and that many share - regarding

\footnotetext{
${ }^{7}$ More precisely: I used decoherence to explain the cat's apparently well-defined state within the alivedead basis, but was careful to say decoherence does not explain why a given observation yields the specific state that it does.
} 
the assumption made in decoherence studies of modeling the system of interest as initially uncorrelated with its environment .

[A]s yet, no technique exists to put a macroscopic oscillator in a pure coherent state... Thus, unlike the cat in Schrödinger's original experiment, the macroscopic mirror cannot start off in a pure state, and in general, will start off in a thermal state. So only a mixture of Schrödinger's cat states can ever be created through unitary evolutions. So the primary aim of Schrödinger's experiment (creating macroscopic superpositions) cannot be achieved this way. However, our aim is to test the decoherence of states of a macroscopic object and not to create a pure Schrödinger's cat state. An important point to realize is that the former can be done without necessarily doing the latter. We shall show that the state of the cavity field (the system on which we actually perform our measurements) at the end of our experiment is the same irrespective of whether the macroscopic object (the movable mirror) coupled to it starts off in a thermal state or a coherent state. This is due to the specific nature of the coupling between the field and the mirror. Thus the mixture of Schrödinger's cat states produced has the same observational consequences as a pure Schrödinger's cat as far as our scheme is concerned. (Bose et al., 1999, pp. 3204-3205)

A general argument of this sort is presented in Anglin et al. (1997), a now-classic theoretical paper on decoherence models. Bose and coauthors continue the passage quoted above as follows:

[I]n Schrödinger's case, the coupling between radioactivity and the cat involved highly nonlinear biological processes. So even a small trigger of radioactive decay was sufficient... to produce as much of a change in a cat as killing it. Can we get such a nonlinear coupling to produce a drastic change in the state of the macroscopic mirror from small changes in the state of the cavity field? The answer to this is that it is really not necessary to have a drastic change in the state of the macroscopic mirror. Even a superposition of macroscopically nondiscernible states is sufficient to produce a detectable rate of decoherence if the the mirror is sufficiently macroscopic. (ibid., p. 3205)

After carrying out the promised calculations, the authors find that the state of the cavity field at $t=2 \pi / \omega_{m}$ (with $\omega_{m}$ the frequency of the oscillating mirror) is entirely independent of the initial thermal state of the oscillating mirror. They summarize (ibid., p. 3207):

This feature... implies that the effects on the cavity field will be the same irrespective of whether the mirror started off in a mixture of coherent states (like a thermal state) or in a single coherent state. This makes the imprint of the demise of a single Schrödinger's cat state on the cavity field identical to the imprint made by the demise of a mixture of several such states. 
In sum, I agree with FL's response to VE: although it is by no means trivial to assume that the cat state is initially uncorrelated, this point has been addressed thoroughly in the literature, both theoretically and experimentally. Of course the strength of this assumption will depend importantly on the details of a particular system-environment interaction and the internal dynamics of both. More on this shall be said below.

\section{Reply to Okon and Sudarsky}

In the remainder of this paper I turn to criticisms raised specifically by OS. The present section discusses seven arguments they make against my thesis in non-relativistic quantum theory. The final section then briefly discusses the challenges OS raise regarding the relativistic applications of my thesis.

\subsection{Defining Decoherence}

After quoting my definition of decoherence, OS describe my language as "poetic", remarking that "[a]lthough one might have some intuitive feeling about things becoming random when they are too complicated, the fact is that the unitary evolution provided by the Schrödinger equation contains absolutely nothing random" (p. 854).

What I state in the original paper is that decoherence occurs only after the initial system has become entangled with an environment, which results in the local (i.e., with respect to the initial system) suppression of unitarity but the continued unitary evolution of the Schrödinger equation globally (i.e., with respect to the new system-environment composite system). I ascribed randomness not to Schrödinger evolution, but to the averaging of the system's phase relations in specific bases due to environmental interactions. Clearly I am not saying the Schrödinger equation itself contains stochasticity.

But leaving such misreadings aside, I took myself to be in the mainstream regarding my usage of concepts like decoherence, coherence and phase relations, but perhaps more clarification is in order. Here again is Bacciagaluppi (2012), defining decoherence in the context of a double-slit experiment:

The phase relation between the two components of the wavefunction, which is responsible for interference, is well-defined only at the level of the larger system composed of electron and stray particles, and can produce interference only in a suitable experiment including the larger system. Probabilities for results of measurements performed only on the electron are calculated as if the wavefunction had collapsed to one or the other of its two components, but in fact the phase relations have merely been distributed over a larger system. It is this phenomenon of suppression of interference through suitable interaction with the environment that we call "dynamical" or "environmental" decoherence. 
Taking a step back, one might ask what is meant by coherence, or coherent superpositions. These terms were inherited from classical optics and mechanics, but one must never forget their origin: an important terminological shift occurs in describing classical systems (e.g., "coherent superposition" as describing higher energy modes in an harmonic oscillator) as opposed to quantum systems. Dirac warns his readers along these lines at the very start of Principles of Quantum Mechanics:

The assumption of superposition relationships between the states leads to a mathematical theory in which the equations that define a state are linear in the unknowns. In consequence of this, people have tried to establish analogies with systems in classical mechanics...which are governed by linear equations and for which, therefore, a superposition principle holds. Such analogies have led to the name "Wave Mechanics" being sometimes given to quantum mechanics. It is important to remember, however, that the superposition that occurs in quantum mechanics is of an essentially different nature from any occurring in the classical theory, as is shown by the fact that the quantum superposition principle demands indeterminacy in the results of observations in order to be capable of a sensible physical interpretation. The analogies are thus liable to be misleading. (Dirac, 1958, p. 14)

He expands on this theme a few pages later:

In the case of a classical system for which a superposition principle holds, for instance a vibrating membrane, when one superposes a state with itself the result is a different state, with a different magnitude of the oscillations. There is no physical characteristic of a quantum state corresponding to the magnitude of the classical oscillations, as distinct from their quality, described by the ratios of the amplitudes at different points of the membrane. Again, while there exists a classical state with zero amplitude of oscillation everywhere, namely the state of rest, there does not exist any corresponding state for a quantum system, the zero ket vector corresponding to no state at all. (ibid., pp. 17-18)

What, then, is quantum coherence (or: what are coherent superpositions in the quantum case)? In a classic introductory text on algebraic quantum field theory, the author provides a proof for the following definition of coherent superpositions:

$[\mathrm{P}]$ ure states are called mutually coherent if, superimposing, they produce again pure states. (Hence, it is clear that coherence and the superposition principle are closely related. In fact, the coherence of a certain set of pure states an the fulfillments of the superposition principle for this set are merely different formulations of the same physical property.) (Horuzhy, 1990, p. 137)

In Blum's textbook on density matrices, an entire section is dedicated to discussion of the "important concept of 'coherent superposition"', wherein the author explains: 
A pure state can always be written as a linear (completely coherent) superposition of basis states. The magnitudes and phases of the coefficients in this expansion are well defined (apart from the overall phase); that is, there exists a definite phase relationship between the basis states. The other extreme is a mixture of independently prepared basis states $\left|\phi_{n}\right\rangle$ represented by the density operator $\rho=\sum_{n} W_{n}\left|\phi_{n}\right\rangle\left\langle\phi_{n}\right|$ without any definite phase relation. $\rho$ is diagonal in the $\left\{\left|\phi_{n}\right\rangle\right\}$ representation and, by definition, the states $\left|\phi_{n}\right\rangle$ overlap incoherently. (Blum, 1996, p. 51)

And here again is Haroche, defining coherence and decoherence for field states: "In general, a field state is described by a density matrix $\rho$, whose diagonal elements $\rho_{n n}$ in the Fock state basis are the $P(n)$ probabilities, and the off-diagonal ones, $\rho n n^{\prime}$, describe the field coherence" (Haroche (2012), pp. 85-86).

Thus, for a density operator

$$
\rho_{m n}(t)=a_{m}(t) a_{n}^{*}(t),
$$

one generally identifies quantum coherence with the off-diagonal matrix elements (i.e., interference terms),

$$
\rho_{m n}=\sum_{j} p_{j}\left(a_{j}\right)_{m}\left(a_{j}\right)_{n}^{*},
$$

whose time-dependent phase factor $e^{-i \omega_{m n} t}$ describes the evolution of coherent superpositions. Because the phases are complex, multiplication of all phase terms by the same factor (itself complex) results in an unchanged state, so what is relevant for maintaining coherence is constancy of the ratio of phase factors. Formally: if the phase difference between two components of a superposition $\delta$ satisfies $\frac{d \delta}{d t}=0$ then the superposition is called coherent.

The sense in which I applied the term "randomized" to a system's phase relations after environmental interaction is also in keeping with standard descriptions of these processes. Two quotes from recent quantum optics textbooks in evidence: on p. 171 of Fox 2006 one finds "...wave function interference can only occur when there is a definite phase relationship... This occurs in the superposition state, but not in a statistical mixture, where the different particle wave functions all have random phases with respect to each other." And from p. 12 of Walls and Milburn 2012: "The coherent states have an indefinite number of photons which allows them to have a more precisely defined phase than a number state where the phase is completely random. The product of the uncertainty in amplitude and phase for a coherent state is the minimum allowed by the uncertainty principle. In this sense they are the closest quantum mechanical states to a classical description of the field."

Finally, OS assume I was mistaken in using the word "commute" instead of "couple" in my definition of decoherence. I was not. In one of the original decoherence papers (a paper I shall discuss in more detail below), Zurek explains: 
The form of the interaction Hamiltonian between the apparatus and its environment is sufficient to determine which observable of the measured quantum system can be considered "recorded" by the apparatus. The basis that contains this record - the pointer basis of the apparatus - consists of the eigenvectors of the operator which commutes with the apparatus-environment interaction Hamiltonian. Thus the environment can be said to perform a nondemolition measurement of an observable diagonal in the pointer basis. (Zurek, 1981, p. 1516)

And again in the body of the paper (ibid., p. 1519):

The apparatus-environment interaction can then be regarded as an additional measurement establishing nonseparable correlations between the apparatus and the environment. ... However, when the [interaction] Hamiltonian $\mathcal{H}_{a s}$ commutes with the observable $\hat{\Pi}$ of the apparatus, then this particular observable will not be perturbed. Only the basis consisting of the eigenstates of $\hat{\Pi}$, the pointer basis, will contain nothing but the information about the quantum system itself. Moreover, the combined $a \mathcal{S}$ system is now represented by a mixture diagonal in a particular product basis, consisting of the eigenvectors of the pointer basis of the apparatus and the corresponding relativity states of the system.

\subsection{Decoherence and the Measurement Problem}

From OS's $\S 2$ (pp. 853-854):

$[\mathrm{D}]$ ecoherence is deemed to solve (at least important parts of) the measurement problem and to explain the quantum-to-classical transition. The idea is that decoherence, which is a straightforward consequence of a purely unitary evolution, is able to effectively explain or bring about the collapse of the wave function, which is, of course, at the root of the measurement problem. Similarly, decoherence is said to dynamically single out a preferred basis which is supposed to coincide with the classical one, thus explaining the emergence of classicality.

They go on to say that the purpose of the section will be "to carefully show that, contrary to widespread believe [sic], decoherence does not help in the resolution of these foundational questions. The main reason being... that in order for decoherence to accomplish what it is supposed to, one needs to assume the very thing that is to be achieved."

The fact that decoherence does not solve the (entire) measurement problem has long been understood. Joos and Zeh write in their foundational decoherence paper (Joos and Zeh, 1985): "Of course no unitary treatment of the time dependence can explain why only one of these dynamically independent components is experiences." And Joos again, in 
his 2000 paper (Joos, 2000, p. 14): "Does decoherence solve the measurement problem? Clearly not. What decoherence tells us, is that certain objects appear classical when they are observed." Extensive references in accord with these sentiments as well as further argumentation are given in the unambiguously titled paper of Adler (2003), "Why decoherence has not solved the measurement problem." I refer unconvinced readers to this review of the debate, and reiterate that this question has widely been considered settled for quite some time now.

The error OS and others make in assuming, contrary to my printed words, that I believe decoherence solves the measurement problem in its entirety, only makes more apparent my motivation for adopting Schlosshauer's deconstruction of the measurement problem. It is incorrect to say decoherence solves the measurement problem, but it is also incorrect to say decoherence does nothing to alleviate certain longstanding questions pertaining to observation. For example, it is now the received view that decoherence explains the existence of preferred bases - and not just in the classical regime (however one defines "classical"). ${ }^{8}$

Hence the importance of separating out the question of general outcomes ("why this set of eigenvalues?") from the question of specific outcomes ("why this particular eigenvalue?"): the former is understood, while the latter is not. However - and herein lies the crux of my original paper - much of the contemporary theoretical work I encountered called upon interpretations of quantum mechanics to solve more than just the problem of specific outcomes. Thus I perceived an unnecessary overlap between the cache of explanatory goods readily available via decoherence and the explanatory work theorists like $\mathrm{VE}$ and OS wanted specific interpretations to do for them. My aim was to disentangle this overlap, and thereby restrict the amount of philosophical baggage being needlessly hauled into relativistic discussions of quantum mechanics. A similar project - philosophical housekeeping regarding the measurement problem - is carried out in Wallace (2012a). Although Wallace's ultimate aim is to defend the Everett interpretation, he too sees the necessity of separating the projection postulate out of the "standard formalism" and recasting the measurement problem to be in closer alignment with experimental practices.

One final, philosophical point: even if, like OS, one does not agree with my particular framing of the measurement problem, all I claimed to do in the original paper was demonstrate that my arguments run successfully given that framing. And this, I insist, I have done.

\footnotetext{
${ }^{8}$ Instead of defending in detail what I name with confidence "the received view", I direct skeptics to the wealth of literature - spanning nearly half a century - supporting this claim. For starters: Zeh (1970), Kübler and Zeh (1973), Zurek (1981), Zurek (1982), Giulini et al. (1995), Giulini et al. (1996), Diósi and Kiefer (2000), Mensky (2000), Zeh (2002), Zurek (2003), Duplantier et al. (2007), Joos (2005), Zurek (2007), Stamp (2006), Castagnino et al. (2007), Janssen (2008), Lombardi et al. (2010), Gell-Mann and Hartle (2014).
} 


\subsection{Decoherence and the Emergence of Classicality}

The second foundational issue OS do not think decoherence can resolve is the emergence of classicality. Their reason has to do with circularity: they claimed that decoherence can only explain the emergence of classicality by assuming "the very thing that is to be achieved" (OS p. 854). It isn't entirely clear what this means. If it is meant to be a criticism of assuming an initially uncorrelated system and environment (as many decoherence models do), I addressed this above in $\S 2.2$. If it is meant to be a criticism of using classical observables in order to analyze decoherence processes where classical behavior results, I addressed this in my original paper in the section on "closing the epistemic circle". If it is meant to be a criticism about decoherence resulting in classical behavior generally - that is, for all systems - this was explicitly not my claim. Indeed, I discuss at some length in the original paper how decoherence and its selection of a preferred basis (and why that basis is typically position for macroscopic objects) is highly sensitive to the details of the system's self-dynamics, the environmental dynamics, the system-environment coupling, and the relative strength of all of these within in the total Hamiltonian.

While FL's contribution to the debate contains a response to the question of emergent classicality with which I agree (although I have niggling worries about their use of the term "objective"), it is somewhat brief and may not hit on the precise sense of circularity that troubles OS. Hence in this section I shall expand on several points made regarding the classical-to-quantum transition in my original paper, starting with remarks about the definition of classicality, and necessary/sufficient conditions for the emergence thereof.

As I have discussed elsewhere in more detail (Crull (2013) and ch. 4 of Crull (2011)), what is understood by classicality or the how one delimits the classical and quantum domains varies. As Joos writes in the introduction to his contribution in Giulini et al. (1996),

It is now increasingly being realized that the conventional treatments of the classical limit are flawed for a simple reason: they do not represent any realistic situation. The assumption of a closed macroscopic system (and thereby the applicability of the Schrödinger equation) is by no means justified in the situations which we find in our present universe. Objects we usually call "macroscopic" are interacting with their natural environment in such a strong manner that they cannot even approximately be considered as isolated, even under extreme conditions. This observation opens up a new approach to the understanding of classical properties within the framework of quantum theory.

Here are but a few definitions of classicality: as Newtonian or quasi-Newtonian motion (characterized by Ehrenfest's theorem), as classical probability distributions or statistical ensembles (characterized by the Liouville regime), as the limit $n \rightarrow \infty$, as the limit

$\hbar \rightarrow 0$, and as mass $\rightarrow 0$. All of these definitions are too crude in and of themselves. That Ehrenfest's theorem provides neither a necessary nor sufficient condition for defining the 
classical regime is the central thesis of Ballentine et al. (1994). One is tempted too heartily in the case of the Liouville regime to interpret probabilities incorrectly: although in certain cases using Liouville's theorem one can recover a probability distribution identical to a classical statistical distribution, formal similarity does not always entail ontological similarity. Indeed, the quantum Liouville equation is a density matrix which, as my critics well know, captures fully the statistics of a system's statespace but cannot substantiate further physical interpretation. Defining classicality as the limit as quantum number $n$ approaching infinity will succeed in some but not all cases. This is proven in Messiah (1965) and Liboff (1984), both of whom draw upon the fact that the uncertainty relations set an insurmountable limit upon how approximately continuous classical energy spectra can become. Neither will the limiting case of decreasing Planck's constant suffice, as this quantum-classical borderline is in many cases a singularity (see Batterman (1995), Batterman (2002), Berry (1994), Berry (2001) and Bokulich (2008) for fuller discussions of the failure of this approach to classicality). The naivety of relying on mass for one's definition of classicality is evident both through examples of massive systems (like Weber bars) that nevertheless behave quantum mechanically, and through macroscopic quantum effects such as circulation quantization in superfluid helium, the Josephson effect in superconducting and, trivially, laser beams.

Other studies of the emergence of classicality employ a blend of the above definitions. One such a study focusing on nonlinear systems can be found in Habib et al. (1998). I quote them at some length below, as they provide not only a comparative study of decoherence versus coarse graining methods for scrutinizing the classical-quantum border, but also helpfully articulate necessary and sufficient criteria for emergent classicality:

A mechanism responsible for the quantum to classical transition should explain not just how expectation values can converge to the same answer, but also lead to compatible effective phase space distributions. A common approach is an appeal to coarse graining, a formal procedure implemented typically by convolving the individual distributions with a Gaussian distribution and then comparing the two resulting coarse-grained distributions. This approach has three defects. First, as a formal mathematical procedure it can always be inverted, and thus offers no physical insight. Second, this coarse graining does not alter the dynamics, and hence cannot improve the convergence of expectation values. Third, for the classical system, the notion of a trajectory is lost and, along with it, the notion of a Lyapunov exponent.

In contrast to the coarse-graining approach, decoherence provides a dynamical explanation of the quantum-to-classical transition by taking into account interactions with an (external or internal) environment of the system - degrees of freedom that effectively monitor and, therefore, select certain stable or "pointer" observables destined to become the classical variables. ... Diffusion terms in these [master] equations automatically coarse grain the distributions, 
not a physical effect of the coupling to the environment rather than a mathematical trick. The degree of coarse graining is determined by the interplay between the dynamics of the system and the nature and strength of the coupling with the environment. Moreover, the effectively classical master equations that describe the postdecoherence dynamics admit a Langevin description of trajectories allowing for the existence of a Lyapunov exponent. We demonstrate below that decoherence dramatically improves the correspondence of the expectation values, leads to the existence of a single phase space distribution, and allows for a Lyapunov exponent to exist at late times. (Habib et al., 1998, p. 4362)

In other words, an approach involving decoherence circumvents precisely those problems arising for coarse graining approaches (on coarse-graining methods cf. FL's paper and references therein). The authors carry out their study by calculating evolution of a driven oscillator in a bosonic field, the dynamics of which are best captured by a quantum Brownian motion decoherence model. They focused on the effects of decoherence in the weak coupling, high temperature limit where dissipation is negligible for early times (and so loss of coherence could not be attributed to energy exchange). Using the Wigner function to illustrate dynamics in this regime, they found that while decoherence suppresses interference terms, "at the same time, noise smooths out the fine structure of the classical distribution in such a way that quantum and classical distributions and expectations values both converge to each other. Thus, one concludes that the decohered quantum evolution does go over to the classical Fokker-Planck limit" (ibid., pp. 4363-4364).

In short, because none of the usual definitions are sufficiently rigorous for explaining classicality in a universal way, discussions of the quantum-to-classical transition must never stray far from detailed information about specific system dynamics and their interactions with specific environments. Since decoherence models are designed to examine precisely these dynamics in wide-ranging situations, many consider decoherence studies the most promising approach to the question of emergent classicality.

To add more grist to the mill, consider briefly the dynamics of three scenarios in which different components of the system-environment total Hamiltonian are negligible: (i) when intrinsic dynamics of both system and environment are negligible with respect to interaction strength $\left(H_{t o t} \approx H_{\text {int }}\right)$; (ii) when the environment is cold, with negligible intrinsic dynamics and little or no perturbative effect on the system $\left(H_{t o t} \approx H_{\text {sys }}\right)$; and (iii) when no component significantly dominates the total Hamiltonian. These considerations will make more plain how decoherence describes not just the emergence of classicality understood as stability in "traditional" bases like position, but likewise explains the emergence of preferred bases in a variety of energy regimes.

(i): The case of negligible system and environment dynamics, $H_{t o t} \approx H_{\text {int }}$. For macroscopic systems, $H_{i n t}$ will typically exhibit strong position dependence owing to prevalence of $1 / r^{2}$ forces. Thus the set of mutually commuting system-environment 
observables are translational, resulting in the relative stability of the position basis (see Zurek $(1981,1982))$.

Here one might object on grounds of circularity: since the $r$ in the interaction Hamiltonian's $1 / r^{2}$-dependency is more accurately described in terms of some further set of quantum variables, the resultant eigenbasis with respect to $r$ is only apparently classical. Furthermore, in order to explain the preference for position in macroscopic cases, one refers to the dynamics of the Hamiltonian. But the Hamiltonian only results in the dynamics it does because we constructed it to do so. That is, if $H_{\text {int }}$ was written down in terms of particular variables, is it any surprise that stability occurs with respect to those variables?

While I agree that there is circularity here, it is not vicious. For one, this criticism does not properly take into account the fact that the interaction Hamiltonian, while it is indeed constructed by hand, is nevertheless valid from an empirical standpoint. Physicists must bite the bullet at some point, and none should be quicker than they to appreciate the complex interplay between theory and experiment essential to scientific progress. For another, although it strikes OS as suspiciously convenient that decoherence explains what we already know to be the case (e.g., the naturalness of using position as the basis of measurement for macroscopic systems is due to quantum dynamics in noisy environments), this is insufficient grounds for claiming this explanation is wrong. The fact that it is convenient doesn't mean it isn't true. Moreoever, I want to suggest that the sheer volume of experimental literature confirming decoherence models in the mesoscopic regime shifts the explanatory onus from decoherence theorists to those opposing it. ${ }^{9}$ More recently, the practicality of performing nested-interferometry experiments measuring macroscopic superpositions has been argued for persuasively by Pepper et al. (2012), Scala et al. (2013), Sekatski et al. (2014) and Wan et al. (2016).

(ii): Dominant intrinsic dynamics, $H_{t o t} \approx H_{\text {sys }}$. When system-environment dynamics are dominated by the system's self-dynamics, eigenstates of the system's Hamiltonian form the pointer basis. This case was first studied in Paz and Zurek (1999), where the authors found that since quantums typically have strongly energy-dependent intrinsic dynamics, it is in the energy basis that such a system will become most effectively localized upon interaction with a relatively calm environment. More will be said about this regime in following sections.

(iii): The intermediate regime. When the total Hamiltonian is not dominated by any single component - $H_{\text {int }}, H_{\text {sys }}$ or $H_{\text {env }}$ - the evolution of the composite system is subject to aspects of both limiting cases presented above, resulting in a situation analogous to quantum Brownian motion. In these cases, $H_{\text {int }}$ describes environmental monitoring of the system's position and momentum (though, note well, decoherence occurs at different rates in each basis). Thus the combined influence of intrinsic dynamics and interaction strength

\footnotetext{
${ }^{9}$ In addition to research from Haroche's Paris team cited above in $\S 2.2$, the Institute of Quantum Optics and Quantum Information in Austria performed a suite of experiments measuring interference patterns with fullerenes: Arndt et al. (1999), Arndt et al. (2002),Brezger et al. (2002),Hackermüller et al. (2003), Hackermüller et al. (2004), Hornberger et al. (2003).
} 
results in minimum uncertainty wave packets tracing out quasi-Newtonian trajectories in the system's phase-space. Experiments proposed and conducted on the basis of the quantum Brownian motion model (and so testing this intermediate regime) include Hornberger (2006), Kokorowski et al. (2001), Monroe et al. (1996), Myatt et al. (2000) and Paz et al. (1993) among many others.

\subsection{Decoherence and EPR States}

On p. 854 OS write: "It could be that what is really meant [by my definition of decoherence] is that the state of a composite system is entangled, then each component does not possess a well-defined state. However, if that is all there is to the loss of coherence, then one must conclude that any entangled system, such as an EPR pair, is not coherent."

Being unable to factorize a composite system (components do not possess well-defined states) is the definition of entanglement, not of decoherence. Though entanglement is certainly prerequisite for decoherence, this does not make them identical processes. The sentence following my definition of decoherence in the original paper makes this clear: "The cause of decoherence processes is entanglement with external degrees of freedom" (Crull, 2015, p. 2021).

Leaving aside this confusion of cause and effect, a charitable interpretation of OS's above statement might be as follows. If entanglement leads to decoherence, and the effect of decoherence is to suppress correlations beyond observability, how is it that correlations are nevertheless measured? To answer this, one must remember that the property of coherence - and therefore decoherence - is basis specific. Furthermore, decoherence occurs at different rates within the initial system depending on the nature of the environmental interaction. While it is indeed true that maximally entangled states (engineered in carefully controlled environments as they must be) maintain coherence with respect to certain preselected bases long enough to be measured, this is not necessarily true for all bases and certainly not true for all time. Were quantum coherence so easily maintained, quantum computing would not face the enormous technical barriers it does.

In Hartmann and Suppes' paper "Entanglement, Upper Probabilities and Decoherence in Quantum Mechanics", the authors calculate the decay of an EPR state under the influence of decoherence as a test measure for their own computation of upper probability measures on decay times of quantum correlations (Hartmann and Suppes, 2010). More plainly, the decoherence time of EPR states is considered the standard against which they evaluate the success of their probability measures on Bell-type states. Consider a typical EPR state,

$$
|\Psi\rangle=\frac{1}{\sqrt{2}}(|01\rangle-|10\rangle),
$$

whose density matrix $\equiv|\Psi\rangle\langle\Psi|$ is

$$
\rho(t)=\left(\frac{1}{\sqrt{2}}|01\rangle-\frac{1}{\sqrt{2}}|10\rangle\right)\left(\frac{1}{\sqrt{2}}\langle 01|-\frac{1}{\sqrt{2}}\langle 10|\right) .
$$


This system is then coupled to a heat bath modeled by a possibly infinite set of independent harmonic oscillators - effectively a bosonic field. Bosonic fields are analogous to environments containing delocalized modes, and so are appropriate for extended-mode environments like collections of photons, phonons and conduction electrons (see Hines and Stamp (2008), p. 543). That this environment is delocalized in the position basis is not due to prior interaction among modes; the individual modes are assumed to be independent of one another and interact only weakly with the system of interest. Assuming independent environmental modes will allow for the construction of a total Hamiltonian wherein the environment's self-Hamiltonian is negligible for broad temperature and frequency ranges. One writes the environment's Hamiltonian as

$$
H_{e n v}=\sum_{k} \frac{1}{2 m} p_{k}^{2}+\frac{1}{2} m \omega^{2} q_{k}^{2},
$$

where individual oscillators $k=1,2,3 \ldots$ are assumed to occupy ground states prior to measurement. The indices have been dropped on oscillator masses $m$ and frequencies $\omega$ as one assumes for simplicity negligible variance in these terms. $p$ and $q$ denote canonical coordinates of the $k$ th oscillator. Because the EPR state considered by Hartmann and Suppes is a spin-1/2 system, the spin-boson model will be most appropriate for calculating decoherence rates, with the added benefit that these models are exactly soluble. The intrinsic dynamics of a spin-1/2 system with finite energy barrier are represented (following Leggett et al. (1987) and Schlosshauer (2007)) by a double-well potential where occupation of the left well (say) effectively describes a spin-up state $|0\rangle$, and occupation of the right well a spin-down state $|1\rangle$. If Pauli matrix $\sigma_{z}$ takes these states as eigenstates, then a spin-1/2 system's Hamiltonian can be expressed

$$
H_{s y s}=-\frac{1}{2} \hbar \Delta_{0} \sigma_{x}+\frac{1}{2} \epsilon \sigma_{z},
$$

with $\epsilon$ characterizing the energy difference between ground states of the potential wells, and $\Delta_{0}$ the tunneling element. In the limiting case of well symmetry, $\epsilon$ will be negligible compared to tunneling element $\Delta_{0}$, giving

$$
H_{E P R}=-\frac{1}{2} \hbar \Delta_{0} \sigma_{x}
$$

The initially uncorrelated interaction Hamiltonian for the spin-boson model (irrespective of whether one has included a tunneling term in the Hamiltonian of the system) is constructed as

$$
H_{\text {int }}=\sigma_{z} \otimes \sum_{k} c_{k} q_{k}
$$

Here the Pauli matrix $\sigma_{z}$ defining the discrete spin basis for the system is linearly coupled to coordinate $q_{k}$ of the $k$ th interacting environmental harmonic oscillator. One expects no 
thermal dissipation due to system-environment interaction in this model: the environment's self-Hamiltonian commutes with neither the system's self-Hamiltonian nor the interaction Hamiltonian, as the latter two contain spin vectors while the environment does not. This is extremely important, as it indicates that the dynamics observed cannot be due to thermal exchange, but solely to decoherence.

The master equation for the spin-boson decoherence model of the reduced density matrix of the system $\rho_{S}(t)$ (a partial trace over environmental degrees of freedom), is the Born-Markov equation:

$$
\begin{aligned}
\frac{d}{d t} \rho_{S}(t)= & -i\left[H_{S}, \rho_{S}(t)\right]- \\
& \int_{0}^{\infty} d \tau\left\{\nu(\tau)\left[X,\left[X(-\tau), \rho_{S}(t)\right]\right]-i \eta(\tau)\left[X,\left\{X(-\tau), \rho_{S}(t)\right\}\right]\right\}
\end{aligned}
$$

where $\nu(\tau)$ is the noise kernel of the environment,

$$
\nu(\tau) \equiv \int_{0}^{\infty} d \omega J(\omega) \operatorname{coth}\left(\frac{\omega}{2 k_{b} T}\right) \cos (\omega \tau),
$$

$\eta(\tau)$ is the dissipation kernel of the environment,

$$
\eta(\tau) \equiv \int_{0}^{\infty} d \omega J(\omega) \sin (\omega \tau)
$$

and spectral density $J(\omega)$ for the bosonic environment is

$$
J(\omega) \equiv \sum_{k} \frac{c_{k}^{2}}{2 m_{k} \omega_{k}} \delta\left(\omega-\omega_{k}\right)
$$

In the EPR system described in Hartmann and Suppes, the master equation becomes (p. 99)

$$
\frac{\partial}{\partial t} \rho(t)=-\frac{k}{2} \sum_{i=1}^{2}\left[\sigma_{+}^{(i)} \sigma_{-}^{(i)} \rho(t)+\rho(t) \sigma_{+}^{(i)} \sigma_{-}^{(i)}-2 \sigma_{-}^{(i)} \rho(t) \sigma_{+}^{(i)}\right],
$$

where $k$ is the damping constant and the raising/lowering operators $\sigma_{+}^{i}, \sigma_{-}^{i}$ are related to the Pauli matrices as $1 / 2\left(\sigma_{x}+i \sigma_{y}\right)$ and $1 / 2\left(\sigma_{x}-i \sigma_{y}\right)$, respectively. The authors find the evolution of the EPR state to be, with normalized time parameter $\tau=k t$,

$$
\rho(\tau)=e^{-\tau} \rho(0)+\left(1-e^{-\tau}\right)|00\rangle\langle 00|,
$$

which, the authors note, means that the system asymptotically approaches the ground state (p. 100). They then calculate the expectation values for operators corresponding to non-vanishing two-particle correlations in state $\rho(\tau)$ and announce, "We see that a 'classical' description of the correlations is possible already after a very short period of time (in units of $k^{-1}$ )" (p. 101). Thus even a maximally entangled state will eventually exhibit decoherence. 


\subsection{Decoherence and Pointer Positions}

On pp. 858-859, OS reformulate the measurement problem and conclude with the following remark: "...the prediction of the theory is that the final state of the measurement is a superposition of different (macroscopic) states for $M$ [the measuring device], which is not what we observe in the lab." And further down p. 859: "What we actually see is the absence of superpositions of $M$ in certain bases."

Here there is much to say, and much has already been said. I will restrict myself to discussion centered on two foundational papers in the decoherence literature - Zeh (1970) and Zurek (1981) - and one more recent paper, Diósi and Kiefer (2000). Near the start of Zeh's original 1970 paper (published in this journal), he writes

When describing the process of measurement as a whole in the framework of quantum theory, it is assumed that the apparatus $M$ can be described by a wave function $\phi_{\alpha}$, the state of the total system $M+S$ obeying the Schrödinger equation... In the case of a general state $\varphi$, the final total state now takes the form

$$
\psi(t)=\sum_{n, \beta} c_{n} u_{\alpha \beta}^{n}(t) \phi_{\beta} \varphi_{n}
$$

[where $\{\phi\}_{n}$ is the set of states corresponding to pointer position $n$ of $\left.M\right]$. It represents a superposition of different pointer positions. This result is said to be in contradiction to the axiom of measurement, because the latter states that the result of measurement is one of the states $\sum_{\beta} u_{\alpha \beta}^{n}(t) \phi_{\beta} \varphi_{n}$. It is of course very unsatisfactory to assume that the laws of nature change according to whether or not a physical process is a measurement. ... If there are two possible pointer positions $\{\phi\}_{n_{1}}$ and $\{\phi\}_{n_{2}}$, any superposition $c_{1} \phi_{n_{1}}+c_{2} \phi_{n_{2}}$ must be a possible state. As such superpositions have never been observed...one should at least find dynamical causes for their nonoccurrence. (Zeh, 1970, p. 70)

Zeh continues to describe the statistical interpretation as one attempt to account dynamically for the nonoccurrence of superposed pointer states, but he finds such a story untenable. The next section of the paper begins thus (ibid., pp. 72-73):

The arguments presented so far were based on the assumption that a macroscopic system (the apparatus of measurement) can be described by a wave function $\phi$. It appears that this assumption is not valid, for dynamical reasons:

If two systems are described in terms of basic states $\phi_{k_{1}}^{(1)}$ and $\phi_{k_{2}}^{(2)}$, the wave function of the total system can be written as $\phi=\sum_{k_{1} k_{2}} c_{k_{1} k_{2}} \phi_{k_{1}}^{(1)} \phi_{k_{2}}^{(2)}$. The case where the subsystems are in definite states $\left(\phi=\phi^{(1)} \phi^{(2)}\right)$ is therefore an exception. Any sufficiently effective interaction will induce correlations. The effectiveness may be measured by the ratios of the interaction matrix elements 
and the separation of the corresponding unperturbed energy levels. Macroscopic systems possess extremely dense energy spectra. The level distances, for example, of a rotator with moment of inertia $1 \mathrm{gcm}^{2}$ are of the order $10^{-42} \mathrm{eV}$, which value may be compared with the interaction between to electric dipoles of $1 e \times c \mathrm{~cm}$ at distance $R, e^{2} \times \mathrm{cm}^{2} / R^{3} \approx 10^{-7}(\mathrm{~cm} / R)^{3} \mathrm{eV}$. It must be concluded that macroscopic systems are always strongly correlated in their microscopic states. ... Since the interactions between macroscopic systems are effective even at astronomical distances, the only "closed system" is the universe as a whole. The assumption of a closed system $M+S$ is hence unrealistic on a microscopic scale.

We continue a similar line of thought as it is picked up by Zurek (1981):

What does, in the real-world apparatuses, determine this apparently unique pointer basis $\left\{\left|A_{p}\right\rangle\right\}$, which records the corresponding relative states $\{|p\rangle\}$ of the system?

Interaction with the environment is the key feature that distinguishes the hereproposed model of the apparatus from the manifestly quantum systems. We argue that the apparatus cannot be observed in a superposition of the pointerbasis states because its state vector is being continuously collapsed [sic]. It is the "monitoring" of the apparatus by the environment which results in the apparent reduction of the wave packet. Correlations between states of the pointer basis and corresponding relative states of the system are nevertheless preserved in the final mixed-state density matrix. (pp. 1516-1517)

Although Zurek uses the unfortunate phrase "continuously collapsed" to describe the decoherence process, his subsequent remarks make abundantly clear that he does not mean collapse in the same manner as collapse theorists mean collapse, as no new physical mechanism is being introduced, and the final state - as he reports - is a mixed one. After giving the density matrix for this final mixed state, he makes a statement that not only shows his understanding of there being no physical collapse involved (at least thus far), but also motivates the division of the measurement problem into a question of general outcomes (related to preferred basis), which decoherence does explain, versus a question of specific outcomes, which decoherence does not explain. He writes, "Hence, even though below we do not face the insoluble question of quantum theory of measurement: 'What causes the collapse of the system-apparatus-environment combined wave function?' we do determine into what mixture the wave function appears to have collapsed" (p. 1517). Notice here Zurek is careful to say that the "collapse" or "reduction" is merely apparent.

To substantiate these claims, Zurek investigates a scenario quite similar to the spin- $1 / 2$ system OS explore in their response (more on which below in §3.6). Zurek considers a reversible Stern-Gerlach setup and calculates the final wave function via interaction of the 
spin-up stream with a bistable atom (the "quantum apparatus", with eigenstates $| \pm\rangle$ and $|\mp\rangle)$ to be

$$
|\Psi\rangle=\{|\uparrow\rangle \otimes(\cos A| \pm\rangle-i \sin A|\mp\rangle)+|\downarrow\rangle \otimes| \pm\rangle\} \otimes|\phi\rangle / \sqrt{2}
$$

where $A$ is the action (a constant value after passage through all three magnets), and $|\phi\rangle$ is the position of the wave packet (a recombined up-stream and down-stream after passage through all three magnets). This is a pure state, indicating that there could not have been a "real" collapse of the wave function (p. 1518). Zurek goes on to show that if one considers the spin and the bistable atom independent systems, "the illusion of a collapse may arise" (op. cit.). He demonstrates this by calculating the wave function in a different basis,

$$
\begin{aligned}
& |+\rangle=(| \pm\rangle+|\mp\rangle) / \sqrt{2}, \\
& |-\rangle=(| \pm\rangle-|\mp\rangle) / \sqrt{2} .
\end{aligned}
$$

This basis yields the wave function, equivalent to (16),

$$
|\Psi\rangle=-i\{(|+\rangle \otimes[|\uparrow\rangle+i|\downarrow\rangle] / \sqrt{2})-(|-\rangle \otimes[|\uparrow\rangle-i|\downarrow\rangle] / \sqrt{2})\} \otimes|\phi\rangle / \sqrt{2} .
$$

As one can see, (19) exhibits maximal system-apparatus correlation even in the new basis. This example recalls to mind the above discussion EPR states.

In Zurek's section III he addresses the exact question raised by OS against me: whence the superpositions expected of pointer states? I tried to explain this in my original paper; I will let Zurek answer this time (Zurek, 1981, p. 1519):

$[\mathrm{T}]$ he pointer basis of the apparatus $a$ is chosen by the form of the apparatusenvironment interaction: It is this basis which contains a reliable record of the state of the system $\mathcal{S}$. This in turn determines uniquely those relative states of the system which are correlated with the apparatus. Moreover, apparatusenvironment correlations do not allow one to observe the aS combination in a superposition. Instead, it becomes a mixture diagonal in the basis constructed from the pointer-basis eigenstates $\left|A_{p}\right\rangle$ and the corresponding relative state of the system.

Finally, a quick look at the question of pointer states in the newer paper of Diósi and Kiefer 2000. For a general density matrix $\rho(t)$, they give the usual Markovian master equation,

$$
\frac{d \rho(t)}{d t}=-\frac{i}{2 m}\left[p^{2}, \rho(t)\right]-\frac{D}{2}[x,[x, \rho(t)]]
$$

(where $D$ represents system-environment interaction) used to describe the common situation of environmental degrees of freedom scattering off of a macroscopic object. This effectively localizes it "by carrying away quantum correlations with the object" (p. 3553). 
The authors note that in the extremely general cases described by (20), the term governing coherent, unitary spreading in momentum "competes" with the second term governing localization. However, these competing terms are "approximately balanced for the "equilibrium width' $\sigma_{0} \approx(D m)^{-1 / 4}$ ", where $\sigma$ denotes the system wave function's characteristic width. "It is then reasonable to conjecture," they write, that " $\sigma_{0}$ will be the characteristic width of the pointer states. This is, in fact, what we shall show by using three different methods, all invoking a principle of robustness" (op. cit.), where they understand robustness as I did: as basis-specific dynamical stability during environmental monitoring. Diósi and Kiefer conclude their study with these remarks:

$[\mathrm{W}] \mathrm{e}$ have demonstrated that three different methods of dynamical robustness lead to an essentially unique local pointer basis in case of Markovian local dynamics. The corresponding pointer states follow the classical trajectories up to a tiny random diffusion. Well-defined stochastic differential equations, known from the theory of quantum state diffusion, govern both the formation and the diffusion of pointer states. These states can thus be used to characterize local quasiclassical properties. The pointer states are not an absolute property of the system in itself, but only characterize certain stability properties with respect to interactions with the environment: They are least sensitive to quantum entanglement, which is why interference terms between them cannot be noticed by local observers. (p. 3555)

\subsection{Decoherence and Spin-1/2 Systems}

In their $\S 2$, OS introduce the simple example of two spin-1/2 daughter particles resulting from decay of a spin-0 particle at rest. Treating one daughter particle as the system of interest and the other as the monitoring environment, OS write the system's reduced density matrix and comment that "according to the attitude [of decoherence proponents] described above, particle 2 must be considered as having a definite value, of either $+1 / 2$ or $-1 / 2$, for its spin along the $z$ axis." They then argue that since the system's reduced density matrix (hereafter RDM) could just as well have been written in an alternate basis, "it is clear that taking such a position in [sic] not viable" (p. 864).

That proponents of decoherence "must" consider the system to have a definite value in virtue of merely having written down its density matrix in some arbitrary basis is demonstrably false not only in general (cf. the just-discussed Stern-Gerlach example from Zurek 1981, who uses a nearly identical argument to explicitly deny that decoherence allows for attribution of definite values before measurement), but it specifically contradicts what I wrote in the original paper. No where did I claim that the apparent definiteness of a measurement outcome was due to the ontic state of affairs prior to measurement. Indeed, it was to caution against precisely this metaphysical misstep - of reading off the formalism claims about reality - that I quoted Bitbol's injunction: "One should realize that choosing a starting point has no ontological implication at all" (Crull, 2015, p. 1030). Writing down 
the system's reduced density matrix in a convenient basis does not commit one to such claims about definite values. The discussion of Cucchietti et al. (2005) in my paper was meant to further underscore this crucial point; it would seem the point bears repeating.

Consider the exactly solvable decoherence model of a spin system interacting with a spin environment. In simple cases it is typical to ignore the system's intrinsic dynamics, as a consequence of which

the eigenstates of the interaction Hamiltonian emerge as preferred pointer states of the system (defined as the ones which are "least perturbed" by the interaction with the environment). Thus the eigenstates of the $\sigma_{z}$ operator... are dynamically selected by the interaction with the environment. Indeed, these states are not perturbed by the interaction while other superpositions rapidly decay into their mixtures. (Cucchietti et al., 2005, p.1)

In cases where the self-Hamiltonian of the system is non-negligible, "pointer states do not coincide with the eigenstates of the interaction Hamiltonian but can range from coherent states for the QBM case to eigenstates of the system's Hamiltonian. Their properties are determined by the interplay between the self-Hamiltonian and the interaction with the environment" (op. cit.).

In parallel with the examples given thus far in Zurek's 1981 paper and OS's spin-1/2 system, Cucchietti and coauthors evaluate decoherence models first while neglecting the system self-Hamiltonian (Case 1), and then while incorporating it (Case 2). They show that the dynamically preferred basis in each case is different. This corroborates the point I and others have made about the degree to which a system's effectively distinguishable states in different bases (owing to the relative stability of those bases) depends on the specific nature of the system-environment interaction.

- Case 1: $H_{S}=0$

The composite system-environment $(\mathcal{S E})$ state at arbitrary time $t$ can be written

$$
\left|\Psi_{\mathcal{S E}}(t)\right\rangle=a|0\rangle\left|\mathcal{E}_{0}(t)\right\rangle+b|1\rangle\left|\mathcal{E}_{1}(t)\right\rangle
$$

assuming at $t=0$ that $\left|\Psi_{\mathcal{S E}}\right\rangle$ is pure and there is no system-environment entanglement. Both of these conditions, the authors note, can be easily relaxed; they chose an initially uncorrelated state to simplify their presentation. The RDM for the system is then

$$
\rho_{\mathcal{S}}=\operatorname{Tr}_{\mathcal{E}}\left|\Psi_{\mathcal{S E}}(t)\right\rangle\left\langle\left.\Psi_{\mathcal{S E}}(t)|=| a\right|^{2} \mid 0\right\rangle\left\langle 0\left|+a b^{*} r(t)\right| 0\right\rangle\left\langle 1\left|+a^{*} b r^{*}\right| 1\right\rangle\left\langle\left. 0|+| b\right|^{2} \mid 1\right\rangle\langle 1|
$$

with decoherence factor $r(t)=\left\langle\mathcal{E}_{1}(t) \mid \mathcal{E}_{0}(t)\right\rangle$. Citing earlier calculations carried out by Zurek, Cucchietti et al. write that because $r(t)$ decays rapidly to zero at $t>0$, the off-diagonal (coherence) terms of the system RDM will in suitably large environments experience negligible fluctuations. In other words, $\rho_{\mathcal{S}}$ becomes rapidly approximately 
diagonalized into the mixture of pointer states $\{|0\rangle,|1\rangle\}$. Their further evaluation of $r(t)$ shows that under reasonable assumptions (e.g. that variance in coupling strengths is finite, that the number $N$ of environmental spins is sufficiently large) this factor decays "quite universally" as approximately Gaussian (ibid., pp. 2-3). In the simple case of equal coupling strengths between the system and various environmental modes as well as identical initial spin states for the environment, the composite state becomes

$$
\left|\Psi_{\mathcal{S E}}(0)\right\rangle=(a|0\rangle+b|1\rangle) \otimes_{k=1}^{N}\left(\alpha_{k}|0\rangle_{k}+\beta_{k}|1\rangle_{k}\right),
$$

which yields a decoherence factor $r(t)=\left(|\alpha|^{2} e^{i g t}+|\beta|^{2} e^{-i g t}\right)^{N}$. The decoherence factor's generic quasi-Guassian behavior can be interpreted, the authors write,

...as a result of the law of large numbers: energies $B_{n}$ of the composite $\mathcal{S E}$ system can be thought of as being the terminal points of an $N$-step random walk. The contribution of the $k$ th spin of the environment to the random energy is $+g$ or $-g$ with probability $|\alpha|^{2}$ or $|\beta|^{2}$ respectively. Therefore the set of all the resulting energies must have an (approximately) Gaussian distribution. (ibid., p. 3)

A similar result obtains given random initial states of the environment (e.g., initial environments who states are not products with those of the system).

Summarizing Case 1, Cucchietti et al. have demonstrated that in the weak coupling limit of the spin-spin decoherence model one effectively recovers the dynamics of a spin system coupled to a bosonic field (cf. $§ 3.4$ above; also see Schlosshauer 2005, pp. 228-229).

- Case 2: $H_{S} \neq 0$

Now the authors consider a total Hamiltonian for the spin-spin decoherence model with nonzero system self-Hamiltonian $(\Delta \neq 0)$ :

$$
H_{t o t}=\Delta \sigma_{x}+\frac{1}{2} \sigma_{z} \otimes \sum_{k=1}^{N} g_{k} \sigma_{z}^{(k)}
$$

Because environmental states $|n\rangle\langle n|$ commute with this Hamiltonian, the evolution operator is given by

$$
U(t)=\prod_{n=0}^{2^{N}-1} I \cos \left(\Omega_{n} t\right)-i \frac{\sigma_{z} B_{n}+\sigma_{x} \Delta}{\Omega_{n}} \sin \left(\Omega_{n} t\right) \otimes|n\rangle\langle n|
$$

with $\Omega_{n}^{2}=\Delta^{2}+B_{n}^{2}$. "The physical interpretation of this result," they write, "is that for every state of the environment $|n\rangle$ the effective dynamics of the system is given 
by a magnetic field $\vec{\Omega}_{n}=\left(\Delta, 0, B_{n}\right)$ in the $X Z$ plane" (ibid., p. 5$)$. The system RDM is then, for arbitrary time $t$,

$$
\rho(t)=\sum_{n=0}^{2^{N}-1}\left|c_{n}\right|^{2} U_{B_{n}}(t) \rho(0) U_{B_{n}}^{\dagger}(t) .
$$

Using this RDM the authors were able to solve the two limiting cases of weak (slow) system self-dynamics and strong system self-dynamics. In the first limit, the authors note that "even a small self-Hamiltonian of the system always ends up turning a fast (Gaussian) decay into a slow (power law) one" (op. cit.), which translates to nontrivially distinct dynamical situations.

Summarizing Case 2, the authors make the following remarks regarding nontrivial intrinsic dynamics (op. cit.).

The states which are dynamically selected by the environment are dramatically different in the two opposite regimes we examined above. For small values of $\Delta$, the eigenstates of the $Z$ component of the central spin are pointer states. They are minimally perturbed by the interaction with the environment (in the previous section, where $\Delta=0$ was assumed, this emerged as an exact result since $p_{z}$ is conserved). However, for large $\Delta \ldots$ the fact that $p_{x}(t \rightarrow \infty) \simeq 1$ is a signature of the decoherence process selecting a completely different set of pointer states. In fact, in this case, the pointer states turn out to be eigenstates of the system Hamiltonian, which is proportional to $\sigma_{x}$.

In addition to the conclusions made in the two cases above, Cucchietti et al. end the paper by emphasizing once more that "by adding a self-Hamiltonian for the system one can dramatically change the main features of the decoherence process" (ibid., p.7).

Returning to OS's spin-1/2 example: Given its origin as a subsystem of an entangled composite, the RDM for particle 2 ("the system") necessarily represents an impure state. Interpreting it as a classical statistical ensemble is a mistake frequently made when the matrix is diagonal in a particular basis, as this renders it formally identical to the density matrix of a proper ensemble. While in the latter case one can (given appropriate knowledge of the system's preparation) interpret diagonalized density matrices as indicating a system's definite occupation of a single pure state from among an ensemble of states, the same cannot be said in cases involving entanglement. While it is indeed correct to say that density matrices represent complete measurement statistics for a given system, this is not the full picture: we also know that an RDM, describing a subregion of composite Hilbert space as it does, is necessarily an improper mixture. No definite values, therefore, can be reasonably imputed to measurement outcomes in such bases. 


\subsection{Decoherence and Observers}

After describing the basic idea of preferred bases from environmental interactions, OS are still bothered by two issues:

The first one is whether the rule given to fix the preferred basis is really observerfree, as advertised. The answer, of course, is that it is not because the division of the world into a system and an environment is totally arbitrary. As a result, different decisions as to how to split a system will lead to different preferred bases. One could try to argue that our epistemic limitations as humans determine which degrees of freedom are accessible to us, and thus dictate a particular way so [sic] select the environment. However, that is very different from claiming, as in the quote [from Schlosshauer] above, that the selection of the basis is observer-free. (pp. 866-867)

In response, I would simply ask OS to reread $\S 2.4$ of my paper and to understand Schlosshauer's remark with more care. In the quote to which OS refer, Schlosshauer is clearly not claiming that the entire process of choosing a measurement basis is observer free - that would be nonsense. Instead, he is saying that the structure of a system-environment interaction Hamiltonian provides "physical, observer-free grounds" motivating choice of such a basis (cf. OS p. 866). In other words: the dynamical emergence of a pointer basis via decoherence is a physical process independent of any observer, and this observer-free process motivates one's choice of basis. Again: despite the mundane fact that experiments are carried out by experimentalists and so are never "observer free", the experimentalist's motivation for carrying out her measurements in a particular basis can be given dynamical underpinning by decoherence.

When one reads Schlosshauer this way, one sees that he is corroborating exactly what OS themselves say, as I originally wrote, and as briefly discussed in FL's contribution to this debate: one of the key lessons from decoherence is that it reveals the profound degree to which division of the world into a system and an environment is arbitrary. The crucial difference between myself and OS is that while they conclude from this arbitrariness that decoherence is not observer free, I conclude that for this reason special ontological status cannot be attributed to any so-defined system or environment, nor indeed to any measurement basis or corresponding set of observables.

Some of the confusion surrounding the concepts of "measurement" and "observer" given decoherence can be cleared up by distinguishing two importantly different scenarios:

- When one is discussing controlled measurements in the laboratory, while it is trivially true that there are observers (defined in the traditional way) and measurements (defined in the traditional way), the choice of measurement basis is grounded in which basis is "more natural" or "more stable" compared to others, and the stability of certain bases in this regard is explained by einselection. There is no better explanation on offer, from within the standard formalism alone, for why different system-environment 
contexts lead to different dynamics (and in some cases different observables); I maintain that anyone who claims otherwise incurs the burden of proof. Nevertheless, speaking of this procedure generally, there are certainly observer-initiated "cuts" made between system, environment, measuring apparatus and observer, and these cuts will (as mentioned before) affect resultant dynamics.

- When one is discussing uncontrolled interactions "in the wild", which degrees of freedom become entangled with and are subsequently monitored by certain other degrees of freedom, and in which basis decoherence then occurs, and on what time scale - these are are all dynamic processes requiring no observer, nor do they require an observer-initiated "cut".

Notice the logical difference between these scenarios. The first expresses sufficient conditions for einselection due to decoherence (and these conditions involve measurements and observers), while the other expresses necessary conditions for decoherence and requires neither observers nor measurements (apart from naturally occurring "monitoring"). Thus when it is claimed that decoherence is observer-free, this is certainly not to be read as meaning observers are never involved, only that they are unnecessary.

Here is Bacciagaluppi (2012) once again, describing yet another sense in which decoherence does not require an observer:

The robustness of the preferred states is related to the fact that information about them is stored in a redundant way in the environment (say, because a Schrödinger cat has interacted with so many stray particles: photons, air molecules, dust). This information can later be acquired by an observer without further disturbing the system (we observe - however that may be interpreted whether the cat is alive or dead by intercepting on our retina a small fraction of the light that has interacted with the cat).

Notice that the decoherence process itself is independent of whether or not one later extracts the redundant information about the initial system from the environment.

Logical confusion also lurks in OS's second issue with the decoherence explanation of preferred bases:

$[T]$ the offered explanation of why it is that we have access to one basis, the preferred one, but not others, crucially depends on the alleged suppression of interference achieved by decoherence as long as the reduced density matrix in question is diagonal. However, we already saw that such a link between diagonality and observability is fallacious. As a result, the fact that the density matrix in one basis is diagonal does not imply that such basis [sic] will be special in any empirically interesting sense. Therefore, even if one were to concede that the selection of the basis is observer-free, one still does not have a satisfactory explanation for the preferred basis. (p. 867) 
As in other parts of their reply, here OS introduce a multiplicity of straw-man positions. First, no one seriously discussing einselection makes the claim that it provides access to one basis but not others. Such a statement is blind to the details of system-environment interactions which, among other things, consider the variable decoherence rates of different bases. For example, in quantum Brownian motion models with weak system-environment coupling, the position basis decoheres more rapidly than momentum, and the momentum basis in turn decoheres far more rapidly than the rate of thermal dissipation. These models, along with other decoherence models, not only tell us which basis is preferred, but they tell us quantitatively the degree to which a basis is robust as compared to others, in the guise of model- and basis-specific decoherence rates. Again, one must refrain from conflating what is experimentally accessible with what there is: decoherence theorists are not claiming that the preferred basis is the only basis in which measurements are possible, merely that the preferred basis is the preferred basis.

Secondly, I cannot make sense of the statement "...as long as the reduced density matrix in question is diagonal." My best guess is that this is a mistake of cart-before-horse: assuming that the decoherence theorists' answer to the problem of the preferred basis begins by finding a diagonalized RDM, when instead one starts from observed dynamics, whose statistics are then codified in the RDM formalism (and in fact can be described using a variety of other formalisms, e.g., decoherence channels, consistent histories or reduced Feynman path integrals. On the latter, see the excellent Mensky 2000).

Thirdly, regarding the link between diagonality and observability: if OS had read my paper carefully, they would have understood that I was making precisely the same point about the non-uniqueness of various diagonal bases. Indeed, as many contributors to the decoherence literature are quick to point out, one can always use Schmidt decomposition to rewrite the system's RDM in a diagonal basis. It just happens that due to system-environment dynamics this basis needn't be Hermitian. Thus those who understand decoherence are well-situated to appreciate the tenuous link between the formalism and the appearance of certain bases as stable, or (putting it another way) the appearance of eigenstates of the preferred basis as having "definite" values.

\subsection{Summary of OS's Main Argument}

At the start of $\S 2.5$ (p. 867), OS restate their overarching argument against me. Below I recast it in premise-conclusion form so that the logic of the text (and, if need be, exactly how/where I have misunderstood their thinking) might be made more perspicuous.

1. "Decoherence is supposed to achieve...the suppression of macroscopic interference" but it fails to do so.

1a. One must assume that the system behaves effectively like a classical mixture. 1b. To verify the system's behavior, one must assume the Born rule. 
2. "Decoherence is supposed to achieve...the selection of a preferred basis" but it fails to do so.

2a. This process is not observer-free "as advertised."

2b. This process depends on suppression of macroscopic interference, which fails (from 1).

3. Given 1 and 2, decoherence "does not solve the measurement problem nor explains [sic] the emergence of classicality."

4. Since "the starting point of [my] criticism of our work is the fact that decoherence solves all the relevant interpretational and conceptual problems we worry about," but this is false (from 3), my criticism does not stand.

The first thing to be said is that OS place themselves in the clear minority by arguing against 1 and 2. That decoherence in some circumstances suppresses macroscopic interference (and in other energy regimes suppresses interference in different bases) is the widely accepted answer to this puzzle. But even if one chooses to disregard the vast literature agreeing with me on this point, OS's argument for why it fails is problematic. Regarding 1a, the entire puzzle of why one doesn't observe macroscopic superpositions (particularly pointer positions) only arises because what one does in fact observe is indistinguishable from the behavior of a classical mixture. No assumptions are required; one merely states what appears to be the case: there is a lack of superpositions at the macroscopic scale, and this cries out for explanation. That $1 b$ is false can already be seen from my discussion of the Born rule. Again, the small word effectively makes all the difference; OS seem blind to this point (as well as many other important qualifications I am careful to make in the original paper), and so have produced yet another straw-man argument. What I and others actually claim is the following: in strong agreement with a variety of confirmed models and an array of experimental results, decoherence causes suppression of macroscopic interference such that certain systems, under certain environmental interactions, after a certain amount of time has elapsed, behave effectively like classical mixtures.

Regarding 2, several quick points can be made. Firstly, whether or not this claim is observer-free has nothing to do with its veracity; thus 2a is irrelevant. Even were it somehow germane to the question of the classical-to-quantum transition, it is as I argued above a misunderstanding of what decoherence theorists are really claiming. In defending this point OS once again state that the process is not observer-free "because the partition of the world into a system and an environment is of course arbitrary" (p. 867). There is murkiness here, and I tried to address it above. The take-home point is this: decoherence is observer-free in the sense that it does not require an observer to occur.

As for $2 \mathrm{~b}$, I have explained in detail above that one should correctly understand decoherence-induced suppression of interference terms as occurring in different bases on 
different time scales, according to different interaction details - not only (or always!) suppressing macroscopic interference terms.

As for 3, I never claimed to be solving the measurement problem tout court. I claimed (again, in line with the literature being generated by experts in the field) that decoherence explains the suppression of interference specifically, and that decoherence explains the appearance of classicality in certain situations.

As for 4, again, what I am supposed to have claimed I did not in fact claim. What I did say in my original paper was much more tempered, and I emphasized the exact scope

of my arguments by reiterating my thesis multiple times. Here are excerpts from just the first three pages:

- In the abstract and on p. 2: that certain problems can be "largely bypassed or dissolved," which is weaker than saying they will all be solved.

- On p. 2: that the early incorporation of a particular interpretation is not necessary, which is importantly different from saying that interpretation can be done away with entirely.

- On p. 3: "there is a way to get further into the physics...without declaring adherence to one interpretation or another", which is not claiming we can do without interpretation.

- On p. 3, in case readers didn't catch it the first few times: "Not only do I argue one can remain interpretation neutral for longer, but I also suggest that work in relativistic domains might benefit greatly by incorporating decoherence phenomena."

- On p. 3, once again with feeling: "I argue decoherence allows us to dissolve (most of) the beast [i.e. the measurement problem] and therefore make progress in the suggested theoretical arena."

\section{Decoherence in Relativistic Quantum Theory}

Thus far I have tried to show how, and to what extent, critics of my original paper misunderstood central points therein. If one reads my arguments in the way they were intended, and with the additional ammunition provided by the preceding expansions upon those themes, then one can better appreciate my arguments regarding three aspects of relativistic quantum theory: the origin of cosmic structure, the problem of time in quantum gravity, and the blackhole information loss paradox. In what follows I focus on the first two questions, and I do so for several reasons. Since OS's criticisms of my relativistic claims are built atop serious misunderstandings of decoherence in non-relativistic quantum theory, much of what they say in this portion of their reply has already been addressed above; nevertheless, OS's weightiest criticisms apply to cosmic structure and the problem of time. 
Furthermore, many of these criticisms overlap with issues raised in connection with the third topic, the information loss paradox. Finally, I have become convinced since the publication of my original paper that while a consensus on the first two questions does seem to be emerging in the literature (and that consensus makes important use of decoherence), the same cannot be said of literature on the information loss paradox. Thus, while I stand by my conclusions, I recognize that my treatment of this paradox in the original paper reflects the unsettled nature of this question more generally, and deserves fuller exposition at a later time.

\subsection{On the Origin of Cosmic Structure}

OS write that the standard account of the universe's transition from a symmetric quantum state without structure to an essentially classical, non-symmetric state with structure, which relies crucially upon quantum fluctuations, is unsatisfactory: "it is easy to show that the standard quantum evolution, via Schrödinger equation [sic], cannot account for the breakdown of the initial symmetry" (p. 869). They argue that objective collapse models, however, "provide an explicit, observer-independent, mechanism for transitions from symmetric to non-symmetric states to occur."

I agree that the account is unsatisfactory, and that is why I urged the incorporation of decoherence dynamics in the first place - as precisely the sort of "explicit, observerindependent mechanism" that could account for the breakdown of this symmetry. OS's primary reason against applying decoherence here is not a new one: if Schrödinger evolution is globally unitary but the relevant system for cosmology is the (closed?) system of the universe, how can decoherence begin?

The question of the universe as a closed system is addressed in FL, but allow me a few further comments. What one must keep in mind is that suitably rich non-thermal dynamics - many degrees of freedom coupling to others with various strengths and for various lengths of times, and so on - such as were present in the early stages of the universe are surely sufficient to transition a symmetric state to an antisymmetric one. For instance, it is known in the decoherence literature that even a single system such as an electron can exhibit decoherence, for example between translational and spin degrees of freedom (see, e.g., Stamp (2006)). OS themselves concede this point somewhat when they treat one part of their maximally entangled pair as the "measuring device" acting upon the other part, the "system". Here the Schrödinger equation is considered to apply to the entangled pair (globally) yet the RDM for the system is computed in two different bases and exhibits local correlations with the "measuring device".

One can easily imagine OS have anticipated this defense when they write the following:

...regardless of the image that the words "quantum fluctuations" can bring to one's mind, and in spite of the fact that different parts of the system may interact and get correlated, the standard story (even including decoherence) is 
incapable of explaining the emergence of structure out of the initially homogeneous and isotropic state. That is, no matter how complicated the internal dynamics may be, the basic assumptions of the standard story guarantee that the state will always be symmetric. (p. 872)

Bacciagaluppi mentions precisely the sort of classical system OS refer to in a footnote to this excerpt, noting that even if a system is in equilibrium, the equilibrium state can be trivially decomposed into a mixture of localized, time-dependent modes whose interactions recover quasi-classical values (Bacciagaluppi (2012)). Zeh also addresses this point when he writes (Zeh, 2003, p. 14): "Entanglement exists, for example, in the static ground state of relativistic quantum field theory, where it is often erroneously regarded as vacuum fluctuations in terms of 'virtual' particles."

In another new paper titled "Quantum decoherence during inflation from gravitational nonlinearities", Nelson use gravitational interactions to construct a minimal mechanism for decoherence, then use this mechanism to approximate the time during inflation when certain modes can be analyzed as classical stochastic variables instead of quantum oscillators(Nelson, 2016, p. 2). The nice thing about Nelson's paper is that it provides a comparison of different approaches to modeling system-environment coupling during the inflationary period (see his $\S 6.2$ ), and instead of using the RDM formalism or employing the usual Lindblad master equation, the author studies evolution of the global wavefunction itself. The paper begins thus:

Recently, it was shown that long-wavelength fluctuations decohere rapidly provided they couple to an environmental sector with an interaction $H_{\text {int }}=\int d^{3} \mathbf{x a}^{\mathbf{3}} \zeta(\mathbf{x}) \mathbf{B}(\mathbf{x})$, where $\zeta$ describes inflaton or curvature fluctuations, and $B$ is a functional of high frequency modes satisfying certain conditions. Here we point out that such a coupling arises from general relativity (GR) and will thus be present in all inflation models. (ibid., p. 2)

By the author's calculations, a super horizon regime is possible in an inflating background, and if such a regime even very minimally couples to a gravitational field, this "is sufficient to decohere vacuum fluctuations and produce classical stochastic perturbations" (ibid., p. 23). "However," the author continues on the next page, "our emphasis is that even if no other interactions arise from the matter sector, decoherence will still occur and arises necessarily as a consequence of gravity. Even in the vanilla case of $V(\phi)=\frac{1}{2} m^{2} \phi^{2}$, the interactions studied here are present." And the concluding point once again (ibid., p. 24): "Beyond single-field, even minimal coupling of matter and gravity will introduce many new couplings of $\zeta$ to additional degrees of freedom, which will lead to an earlier time of decoherence."

A 1995 paper by Ridderbos provides a comparison between a decoherence approach to the emergence of classicality in cosmology and a coarse-graining approach. While these methods, which are both frequently used in the quantum cosmology literature "to make 
the wave function satisfy the necessary conditions for predictions", are phenomenologically equivalent in certain cases, Ridderbos argues that "considerations about the conceptual justifications for these methods lead us to reject the coarse graining method in favour of the decoherence method" (Ridderbos, 1999, p. 42). In a similar vein, researchers like Koksma et al. (2010), Lombardo and Mazzitelli (1996) and Mavromatos and Sarkar (2007) continue to find fascinating results in quantum field theory and cosmology from grounds of decoherence processes. One should be wary of dismissing too quickly this increasing volume of new and interesting work, and in part my original paper intended to draw attention to this ever-broadening avenue of relativistic applications of decoherence.

As a final point, notice that no where in the decoherence account of the origin of cosmic structure is human intervention assumed or invoked. Thus it is unfounded for OS to say of my arguments, as they do on p. 873, that "a human act is supposed to be, at least in part, the cause for the breakdown of the symmetry that leads to the emergence of galaxies, planets and, eventually, humans."

\subsection{On Time in Quantum Gravity}

On p. 875, after reviewing the criticism I originally made of OS's introduction of nonunitary terms, they write: "Apparently, Crull believes that when a system is interacting, it does not evolve in accordance with Schrödinger's equation."

As I argue in my original paper, and in keeping with the consensus view among decoherence specialists, the ubiquity of quantum interactions leads to high probabilities of entanglement. Once entangled, it is the composite system that follows unitary evolution in accordance with the Schrödinger equation, not the subsystem. Hence, my statements about local dynamics not preserving unitary evolution are qualified. And, as I stressed throughout the original paper, because no physical collapse happens on this account (at least not within the time scale on which decoherence is effective), of course I am not claiming that decoherence "brings about a fundamental breakdown of unitarity" (emphasis original), as accused by OS. I did say that decoherence has the potential to provide a detailed picture of the dynamics under effective nonunitarity, and that effective dynamics of this kind were sufficient for achieving the requisite theoretical results.

Why effectively non-unitary dynamics are considered "rather unhelpful" by OS (p. 878) is mysterious to me. I took "effective" to mean something along the lines of being able to reproduce expected results with sufficient accuracy in situations where the underlying picture is assumed to be more complex. In any case, the point here, as before, is that if decoherence effectively gets us a conciliatory dynamical picture, then that is enough. One no longer need to postulate as-yet unobserved physical components (e.g. collapse mechanisms) in order to forge ahead in some measure.

OS conclude their section by restating the commonly-held belief that decoherence will encounter serious problems when applied globally - that is, to the universe as a whole. My argument does not require that the universe undergo a generic or uniform decoherence 
process in the same basis, or at the same rate, or even among the same degrees of freedom. This is very much in line with a brief suggestion made by Bacciagaluppi (2012):

In the context of decoherence theory, one can construct toy models in which the analogue of the Wheeler-DeWitt wave function decomposes into non-interfering components (for a suitable sub-system) each satisfying a time-dependent Schrödinger equation, so that decoherence appears in fact as the source of time.

Even since my original paper, the possibility of bringing decoherence dynamics to bear in understanding various problems of time (including the arrow of time) has been taken up by a number of physicists and the literature has blossomed.

\section{Conclusion}

In this article I have addressed criticisms made of my 2015 paper, most especially those of Okon and Sudarsky. I hope that the more detailed explanations, expansion of literature and increased formal representation given to my original thesis here will prove fruitful for future discussions on the topic of decoherence.

\section{Acknowledgements}

I'd like to thank Max Schlosshauer, Don Howard, Claus Kiefer, Carlo Rovelli, Guido Bacciagaluppi and an anonymous referee for their support and helpful discussions.

\section{References}

Adler, S. L. (2003). Why decoherence has not solved the measurement problem: A response to P.W. Anderson. Studies in the History and Philosophy of Modern Physics 34B, 135142.

Anglin, J., J. Paz, and W. Zurek (1997, June). Deconstructing decoherence. Physical Review A 55(6), 4041-4053.

Arndt, M., O. Nairz, J. Vos-Andreae, C. Keller, G. van der Zouw, and A. Zeilinger (1999). Wave-particle duality of $\mathrm{C}_{60}$ molecules. Nature 401, 680-682.

Arndt, M., O. Nairz, and A. Zeilinger (2002). Interferometry with macromolecules: Quantum paradigms tested in the mesoscopic world. In R. Bertlmann and A. Zeilinger (Eds.), Quantum [Un]Speakables: From Bell to Quantum Information, pp. 333-351. Berlin: Springer. 
Bacciagaluppi, G. (2012). The role of decoherence in quantum mechanics. The Stanford Encyclopedia of Philosophy (Winter 2012 Edition), Edward N. Zalta (ed.), URL = http://plato.standford.edu/archives/win2012/entries/qm-decoherence/.

Ballentine, L., Y. Yang, and J. Zibin (1994). Inadequacy of Ehrenfest's theorem to characterize the classical regime. Physical Review A 50, 2854-2859.

Batterman, R. (1995). Theories between theories: asymptotic limiting intertheoretic relations. Synthese 103, 171-201.

Batterman, R. (2002). The Devil in the Details: Asymptotic Reasoning in Explanation, Reduction and Emergence. Oxford: Oxford University Press.

Bernu, J., S. Deleglise, C. Sayrin, S. Kuhr, I. Dotsenko, M. Brune, J.-M. Raimond, and S. Haroche (2008, Oct). Freezing coherent field growth in a cavity by the quantum zeno effect. Physical Review Letters 101, 180402(4).

Berry, M. (1994). Asymptotics, singularities and the reduction of theories. In D. Prawitz, B. Skyrms, and D. Westerstahl (Eds.), Logic, Methodology and Philosophy of Science IX. Amsterdam: Elsevier.

Berry, M. (2001). Chaos and the semiclassical limit of quantum mechanics (is the moon there when somebody looks?). In R. Russell, P. Clayton, K. Wegter-McNelly, and J. Polkinghorne (Eds.), Quantum Mechanics: Scientific Perspectives on Divine Action. Vatican Observatory: CTNS Publications.

Bertet, P., A. Auffeves, S. Maioli, T. Osnaghi, T. Meunier, M. Brune, J.-M. Raimond, and S. Haroche (2002). Direct measurement of the Wigner function of a one-photon Fock state in a cavity. Physical Review Letters 89, 200402.

Blum, K. (1996). Density Matrix Theory and Applications (2nd ed.). New York, London: Plenum Press.

Bokulich, A. (2008). Reexamining the Quantum-Classical Relation: Beyond Reductionism and Pluralism. Cambridge: Cambridge University Press.

Bose, S., K. Jacobs, and P. Knight (1999). Scheme to probe the decoherence of a macroscopic object. Physical Review A 59(5), 3204-3210.

Brezger, B., L. Hackermüller, S. Uttenthaler, J. Petschinka, M. Arndt, and A. Zeilinger (2002). Matter-wave interferometer for large molecules. Physical Review Letters 88, 100404 .

Castagnino, M., R. Laura, and O. Lombardi (2007, Dec). A general conceptual framework for decoherence in closed and open systems. Philosophy of Science 74, 968-980. 
Crull, E. (2011, May). Quantum Decoherence and Interlevel Relations. Ph.D. Thesis, University of Notre Dame, Notre Dame, Indiana.

Crull, E. (2013). Exploring philosophical implications of quantum decoherence. Philosophy Compass 8(9), 875-885.

Crull, E. (2015). Less interpretation and more decoherence in quantum gravity and inflationary cosmology. Foundations of Physics 45 (9), 1019-1045.

Cucchietti, F., J. Paz, and W. Zurek (2005). Decoherence from spin environments. Physical Review A 72(5), 052113(8).

Davidovich, L., M. Brune, J.-M. Raimond, and S. Haroche (1996). Mesoscopic quantum coherences in cavity QED: Preparation and decoherence monitoring schemes. Physical Review A 53, 1295.

Deléglise, S., I. Dotsenko, C. Sayrin, J. Bernu, M. Brune, J.-M. Raimond, and S. Haroche (2008, Sept). Reconstruction of non-classical cavity field states with snapshots of their decoherence. Nature 455, 510-514.

Diósi, L. and C. Kiefer (2000). Robustness and diffusion of pointer states. Physical Review Letters 85(17), 3552-3555.

Dirac, P. (1958). The Principles of Quantum Mechanics (4th ed.). Oxford: Clarendon Press.

Duplantier, B., J.-M. Raimond, and V. Rivasseau (Eds.) (2007). Quantum Decoherence, Volume 48 of Poincaré Seminar 2005. Birkhäuser Verlag.

Fortin, S. and O. Lombardi (2017). Interpretation and decoherence: a contribution to the debate Vassallo \& Esfeld vs Crull. Forthcoming in Foundations of Physics.

Fox, M. (2006). Quantum Optics: An Introduction. Oxford: Oxford University Press.

Gell-Mann, M. and J. B. Hartle (1996). Equivalent sets of histories and multiple quasiclassical realms. arXiv:gr-qc/9404013v3.

Gell-Mann, M. and J. B. Hartle (2014). Adaptive coarse graining, environment, strong decoherence, and quasiclassical realms. Physical Review A 89(5), 052125(10).

Giulini, D., E. Joos, C. Kiefer, J. Kupsch, I.-O. Stamatescu, and H. Zeh (1996). Decoherence and the Appearance of a Classical World in Quantum Theory (1st ed.). Berlin: SpringerVerlag.

Giulini, D., C. Kiefer, and H. Zeh (1995, April). Symmetries, superselection rules, and decoherence. Physics Letters A 199(5-6), 291-298. 
Habib, S., K. Shizume, and W. Zurek (1998). Decoherence, chaos, and the correspondence principle. Physical Review Letters 80, 4361-4365.

Hackermüller, L., K. Hornberger, B. Brezger, A. Zeilinger, and M. Arndt (2004, Feb). Decoherence of matter waves by thermal emission of radiation. Nature 427, 711-714.

Hackermüller, L., S. Uttenthaler, K. Hornberger, E. Reiger, B. Brezger, A. Zeilinger, and M. Arndt (2003). Wave nature of biomolecules and fluorofullerenes. Physical Review Letters 91, 090408.

Halliwell, J. (1995). A review of the decoherent histories approach to quantum mechanics. Annals of the New York Academy of Sciences 755, 726-740.

Haroche, S. (2012, 8 December 2012). Controlling photons in a box and exploring the quantum to classical boundary. nobelprize.org.

Hartmann, S. and P. Suppes (2010). Entanglement, upper probabilities and decoherence in quantum mechanics. In M. Suárez, M. Dorato, and M. Rédei (Eds.), EPSA Philosophical Issues in the Sciences, Berlin, pp. 93-103. Springer.

Hines, A. P. and P. Stamp (2008). Decoherence in quantum walks and quantum computers. Canadian Journal of Physics 86, 541-548.

Hornberger, K. (2006, Aug). Master equation for a quantum particle in a gas. Physical Review Letters 97, 060601(4).

Hornberger, K., S. Uttenthaler, B. Brezger, L. Hackermüller, M. Arndt, and A. Zeilinger (2003, April). Collisional decoherence observed in matter wave interferometry. Physical Review Letters 90(16), 160401(4).

Horuzhy, S. (1990). Introduction to Algebraic Quantum Field Theory. Dordrecht: Kluwer Academic Publishers.

Janssen, H. (2008). Reconstructing reality: Environment-induced decoherence, the measurement problem, and the emergence of definiteness in quantum mechanics. philsciarchive.pitt.edu.

Joos, E. (2000). Introduction. In P. Blanchard, D. Giulini, E. Joos, C. Kiefer, and I.-O. Stamatescu (Eds.), Decoherence: Theoretical, Experimental, and Conceptual Problems, Proceedings of the Bielefeld Workshop, Nov. 1998, Berlin, pp. 1-17. Springer.

Joos, E. (2005). Dynamical consequences of strong entanglement. In B. Duplantier, J.M. Raimond, and V. Rivasseau (Eds.), Quantum Decoherence, pp. 177-192. Birkhäuser Verlag. 
Joos, E. and H. Zeh (1985). The emergence of classical properties through interaction with the environment. Zeitschrift für Physik B 59, 223-243.

Kokorowski, D. A., A. D. Cronin, T. D. Robers, and D. E. Pritchard (2001, March). From single- to multiple-photon decoherence in an atom interferometer. Physical Review Letters 86(11), 2191-2195.

Koksma, J., T. Prokopec, and M. G. Schmidt (2010). Decoherence in an interacting quantum field theory: The vacuum case. Physical Review D 81, 065030.

Kübler, O. and H. Zeh (1973). Dynamics of quantum correlations. Annalen der Physik 76, 405-418.

Leggett, A., S. Chakravarty, A. Dorsey, M. Fisher, A. Garg, and W. Zwerger (1987, Jan). Dynamics of the dissipative two-state system. Reviews of Modern Physics 59(1), 1-85.

Liboff, R. (1984). The correspondence principle revisited. Physics Today 37, 50-55.

Lombardi, O., S. Fortin, M. Castagnino, and J. S. Ardenghi (2010). Compatibility between environment-induced decoherence and the modal-Hamiltonian interpretation of quantum mechanics.

Lombardo, F. and F. Mazzitelli (1996). Coarse graining and decoherence in quantum field theory. Physical Review D 53(4), 2001-2011.

Mavromatos, N. and S. Sarkar (2007). Probing models of quantum decoherence in particle physics and cosmology. Journal of Physics Conference Series 67, 012011.

Mensky, M. B. (2000). Quantum Measurements and Decoherence: Models and Phenomenology. Dordrecht, Boston \& London: Kluwer Academic Publishers.

Messiah, A. (1965). Quantum Mechanics, Volume I and II. Amsterdam: North-Holland Publishing.

Monroe, C., D. Meekhof, B. E. King, and D. J. Wineland (1996). A 'Schrödinger cat' superposition state of an atom. Science 272, 1131.

Myatt, C. J., B. E. King, Q. Turchette, C. A. Sackett, D. Kielpinski, W. M. Itano, C. Monroe, and D. J. Wineland (2000, 20 Jan). Decoherence of quantum superpositions through coupling to engineered reservoirs. Nature 403, 269-273.

Narozhny, N., J. Sanchez-Mondragon, and J. Eberly (1981). Coherence versus incoherence: Collapse and revival in a simple quantum model. Physical Review A 23, 236-247.

Nelson, E. (2016, March). Quantum decoherence during inflation from gravitational nonlinearities. Journal of Cosmology and Astroparticle Physics 2016, 022. 
Okon, E. and D. Sudarsky (2016). Less decoherence and more coherence in quantum gravity, inflationary cosmology and elsewhere. Foundations of Physics 46(7), 852-879.

Paz, J., S. Habib, and W. Zurek (1993). Reduction of the wave packet: Preferred observable and decoherence time scale. Physical Review D 47, 488-501.

Paz, J. and W. Zurek (1999). Quantum limit of decoherence: Environment induced superselection of energy eigenstates. Physical Review Letters 82, 5181-5185.

Pepper, B., R. Ghobadi, E. Jeffrey, C. Simon, and D. Bouwmeester (2012). Optomechanical superpositions via nested interferometry. Physical Review Letters 109(2), 023601(5).

Raimond, J.-M., M. Brune, and S. Haroche (2001). Manipulating quantum entanglement with atoms and photons in a cavity. Reviews of Modern Physics 73, 565.

Raimond, J.-M. and S. Haroche (2005). Monitoring the decoherence of mesoscopic quantum superpositions in a cavity. Séminar Poincaré 2, 25-64.

Ridderbos, K. (1999). The loss of coherence in quantum cosmology. Studies in the History and Philosophy of Modern Physics 30(B), 41-60.

Rosaler, J. (2015). Is de Broglie-Bohm theory specially equipped to recover classical behavior?ohm theory specially equipped to recover classical behavior? Philosophy of Science 82(5), 1175-1187.

Scala, M., M. Kim, G. Morely, P. Barker, and S. Bose (2013). Matter-wave interferometry of a levitated thermal nano-oscillator induced and probed by a spin. Physical Review Letters $111(18), 180403(5)$.

Schlosshauer, M. (2005). Decoherence, the measurement problem, and interpretations of quantum mechanics. Reviews of Modern Physics 76(4), 1267-1305.

Schlosshauer, M. (2007). Decoherence and the Quantum-to-Classical Transition (2nd ed.). Berlin: Springer.

Sekatski, P., M. Aspelmeyer, and N. Sangouard (2014). Macroscopic optomechanics from displaced single-photon entanglement. Physical Review Letters 112(8), 080502(5).

Stamp, P. (2006). The decoherence puzzle. Studies in the History and Philosophy of Modern Physics 37, 467-497.

Vassallo, A. and M. Esfeld (2015). On the importance of interpretation in quantum physics: A reply to Elise Crull. Foundations of Physics 45(12), 1533-1536.

Wallace, D. (2012a). Decoherence and its role in the modern measurement problem. Philosophical Transactions of the Royal Society A 370, 4576-4593. 
Wallace, D. (2012b). The Emergent Multiverse: Quantum Theory according to the Everett Interpretation. Oxford: Oxford University Press.

Walls, D. and G. Milburn (2012). Quantum Optics. Berlin: Springer.

Wan, C., M. Scala, S. Bose, C. Frangeskou, A. A. Rahman, G. Morely, P. Barker, and M. Kim (2016). Tolerance in the Ramsey interference of a traped nanodiamond. Physical Review Letters 93(4), 043852(8).

Zeh, H. (1970). On the interpretation of measurement in quantum theory. Foundations of Physics 1, 69-76.

Zeh, H. (2002). Ch 2: Basic concepts and their interpretation. quant-ph/9506020v3.

Zeh, H. (2003). There is no "first" quantization. Physics Letters A 309, 329-334.

Zurek, W. (1981). Pointer basis of quantum apparatus: Into what mixture does the wave packet collapse? Physical Review D 24, 1516-1525.

Zurek, W. (1982). Environment-induced superselection rules. Physical Review D 26, 1862 1880 .

Zurek, W. (2003). Decoherence, einselection, and the quantum origins of the classical. Reviews of Modern Physics 75, 715-775.

Zurek, W. (2007). Decoherence and the transition from quantum to classical- revisited. In B. Duplantier, J.-M. Raimond, and V. Rivasseau (Eds.), Quantum Decoherence, pp. 1-32. Birkhäuser Verlag. 